\title{
Tratamiento endoscópico de las epistaxis posteriores. Experiencia del Hospital Clínico de la Universidad de Chile entre los años 2007 y 2011
}

\author{
Endoscopic treatment of posterior epistaxis. Experience at the Hospital Clínico \\ de la Universidad de Chile between the years 2007 and 2011
}

\author{
Eugenio Alzérreca A², Karen León S³ $^{3}$ Paul Boettiger B', Alfredo Naser G'.
}

\begin{abstract}
RESUMEN
Introducción: La epistaxis corresponde al motivo de consulta de urgencia más frecuente en otorrinolaringología. Su etiología es multifactorial, predominantemente de causa traumática. Una alternativa de manejo quirúrgico en los casos de epistaxis significativa es la cauterización o ligadura endoscópica de la arteria esfenopalatina (AEP).

Objetivos: Este estudio pretende revisar la experiencia del Hospital Clínico de la Universidad de Chile en el manejo endoscópico de pacientes con epistaxis posterior.

Material y método: Se revisaron los registros quirúrgicos del servicio entre los años 2007 y 2011 considerando todos los pacientes que fueron tratados por epistaxis posterior mediante cirugía endoscópica nasal.

Resultados: Se revisó un total de 23 casos. La edad promedio de los pacientes fue $52 \pm 18$ años. Sesenta por ciento presentaba el antecedente de hipertensión arterial en tratamiento. Se realizó ligadura con clips más electrocauterización de AEP en 52,1\% de los casos, ligadura con clips de AEP en 26,2\%, y electrocauterización de AEP en 21,7\% de los casos.

Discusión y conclusiones: El manejo endoscópico de las epistaxis posteriores es una técnica sencilla y reproducible, constituyendo una herramienta terapéutica segura y eficaz para estos pacientes.

Palabras claves: Epistaxis posterior, ligadura endoscópica, arteria esfenopalatina, cirugía endoscópica nasal.
\end{abstract}

\begin{abstract}
Introduction: Epistaxis is by far the most common emergency complaint in otolaryngology. Its etiology is multifactorial, predominantly by traumatic causes. An alternative in surgical management of significant epistaxis cases, is the endoscopic cauterization or ligature of the sphenopalatine artery (SPA).

Aim: This study aims to review the experience of the Hospital Clínico de la Universidad de Chile in the endoscopic management of patients with posterior epistaxis.
\end{abstract}

\footnotetext{
${ }^{1}$ Servicio de Otorrinolaringología. Hospital Clínico Universidad de Chile

${ }^{2}$ Médico Cirujano. Magíster en Derecho de la Salud.

${ }^{3}$ Interno Medicina. Universidad de Chile.
} 
Material and methods: The surgical records have been reviewed between the years 2007 and 2011, considering all patients who were treated for posterior epistaxis, by endoscopic nasal surgery.

Results: $A$ total of 23 cases were reviewed. The average age of patients was $52 \pm 18$ years. $60 \%$ of patients had a history of hypertension in treatment. Clip ligature with electrocauterization of the SPA was performed in $52.1 \%$ of cases, clip ligature of the SPA in $26.2 \%$, and isolated electrocauterization of the SPA in $21.7 \%$ of cases.

Discussion and conclusions: Endoscopic treatment of posterior epistaxis is a simple and reproducible technique, being a safe and effective therapeutic tool for these patients.

Key words: Posterior epistaxis, endoscopic ligature of sphenopalatine artery, Endoscopic nasal surgery.

\section{INTRODUCCIÓN}

La epistaxis, definida como un episodio de sangrado activo por la nariz, corresponde al motivo de consulta en urgencia más frecuente en otorrinolaringología. Se estima que aproximadamente el $60 \%$ de la población sufre algún cuadro de epistaxis en su vida, aunque sólo el $6 \%$ de ellos requerirá atención médica para tratamiento y control hemostático, siendo hospitalizados para tal efecto sólo 1,6 por cada 100.000 pacientes ${ }^{1}$. Su incidencia varía con la edad, presentando una distribución bimodal con un peak en niños y jóvenes, y otro en adultos mayores (45-65 años)².

La causa de las epistaxis es multifactorial, y resulta de la interacción de una serie de factores ambientales, locales o sistémicos que afectan la mucosa nasal y los vasos sanguíneos ${ }^{3}$. Frecuentemente, como resultado de causas traumáticas 0 mecánicas, la epistaxis puede progresar a ser incontrolable, o sea aquella hemorragia significativa que requiere asistencia médica para su control. En casos de epistaxis persistente, el rápido y adecuado manejo de la condición inicial del paciente es importante para minimizar la morbilidad y la mortalidad ${ }^{4}$.

Existen diversas estrategias de enfrentamiento, que en general se clasifican en quirúrgicas y no quirúrgicas, las cuales van desde el taponamiento nasal anterior y posterior, electrocauterización, cauterización química y el uso de vasoconstrictores, hasta métodos más complejos utilizados en casos refractarios, como ligadura de vasos, cirugía endoscópica y procedimientos de radiología intervencional (embolización selectiva) ${ }^{5}$.

La epistaxis severa ha sido tradicionalmente tratada con técnicas de taponamientos nasales, sin embargo avances recientes en técnicas endoscópicas e instrumentos para la ligadura segura de las principales arterias que irrigan la mucosa nasal, han logrado mejorar el pronóstico de los pacientes a través de la disminución de hospitalizaciones prolongadas y morbilidades asociadas al taponamiento ${ }^{2,3}$. El control del sangrado con cauterización es uno de los métodos más simple, costo-efectivo y sencillo de realizar en forma endoscópica, pero requiere la identificación precisa de los puntos de sangrado.

En los últimos años, varias publicaciones sugieren como alternativa en el manejo de pacientes con epistaxis posterior, el control quirúrgico de la AEP o sus ramas mediante ligadura bajo visión endoscópica. Estos estudios han mostrado que tanto la ligadura como la cauterización arterial tienen una eficacia y un perfil costo-efectividad similar y bastante aceptable para la resolución de las epistaxis posteriores ${ }^{6-8}$. Otra alternativa existente, reportada por algunos autores como tratamiento ante el fracaso en el manejo inicial, es la embolización selectiva de la AEP o sus ramas, con una eficacia reportada entre $75 \%$ y $83 \%{ }^{9}$.

\section{OBJETIVOS}

En este estudio, se pretende revisar la experiencia del Hospital Clínico de la Universidad de Chile (HCUCh) en el manejo endoscópico de pacientes con epistaxis posterior, con el objetivo de desarrollar nuevos protocolos de tratamiento quirúrgico eficaces y seguros, disminuyendo la morbilidad inherente, la estadía hospitalaria y costos.

\section{MATERIAL Y MÉTODO}

\section{Características generales}

Se realizó un estudio de tipo retrospectivo, descriptivo y transversal en el Servicio de Otorrinolaringología del 
HCUCh. Se revisaron los registros quirúrgicos del servicio entre los años 2007 y 2011, incluyendo en la revisión aquellos casos de pacientes con epistaxis, que fueron tratados mediante cirugía endoscópica de nariz y cavidades paranasales (CPN). Las variables fueron descritas mediante promedios con sus respectivas desviaciones estándar (DE) o frecuencias porcentuales.

Se consideró para la revisión de fichas clínicas, datos epidemiológicos de los pacientes como edad, sexo y comorbilidades, más otros datos de interés como la modalidad de ingreso al hospital y su presentación clínica. Con respecto al tratamiento endoscópico de la epistaxis, se revisó el tiempo transcurrido entre la hospitalización y manejo quirúrgico, el tiempo de duración de la cirugía, la presencia o no de sangrado activo durante el procedimiento, el tipo de técnica para el control de la epistaxis utilizada y la estadía hospitalaria. Se incluyó además información sobre las complicaciones descritas tanto en el procedimiento como en el posoperatorio de los pacientes.

Se aplicó como criterio de exclusión general para el manejo endoscópico de las epistaxis el antecedente de coagulopatía y uso de terapia anticoagulante oral. En todos los casos revisados se obtuvo el consentimiento informado previo a la cirugía realizada y se explicaron las alternativas de tratamiento a pacientes y familiares.

\section{Técnica quirúrgica}

Todos los pacientes fueron tratados inicialmente con taponamiento nasal prequirúrgico. Los procedimientos fueron efectuados bajo anestesia general en pabellón en todos los casos. Se infiltró en forma submucosa con solución de lidocaína al $2 \%$ más adrenalina 1:100.000 a nivel de pared lateral del meato medio, inferior a la lamela basal del cornete medio. En algunos pacientes se realizó uncinectomía o antrostomía para facilitar el abordaje. Posteriormente, el cornete medio fue medializado para acceder al meato medio, donde a continuación se realizó una incisión mucosa en forma de "L" de $15 \mathrm{~mm}$, posteroinferior a la bula etmoidal y 1 $\mathrm{cm}$ anterior a la inserción posterior del cornete medio, continuando hasta la inserción del cornete inferior. A continuación se confeccionó un flap mucoperióstico, el cual es levantado en dirección posterosuperior, hasta que el foramen esfenopalatino, por donde emerge la $A E P$, se hace visible ya sea anteroinferior 0 posterosuperior a la inserción lateral de la cola del cornete medio. Tras localizar la AEP y disecarla con cuidado, se procedió a su cauterización con pinza bipolar o ligadura con clips endoscópicos. Tras ello, se repuso el colgajo mucoperióstico y se dejó taponamiento nasal1 ${ }^{10}$. (Figura 1).

\section{RESULTADOS}

\section{Características prequirúrgicas}

Se revisó un total de 23 fichas clínicas de pacientes con diagnóstico de epistaxis posterior, manejados en el Servicio de ORL del HCUCh, mediante cirugía endoscópica de nariz y CPN. De estos casos, 15 correspondieron a pacientes de sexo masculino $(65,2 \%)$, siendo el resto (8 pacientes) de sexo femenino. La edad promedio de los pacientes incluidos en el estudio fue de $52 \pm 18$ años (rango entre 16 y 81 años). En cuanto a las principales comorbilidades de los pacientes, $60 \%$ presentaba hipertensión arterial

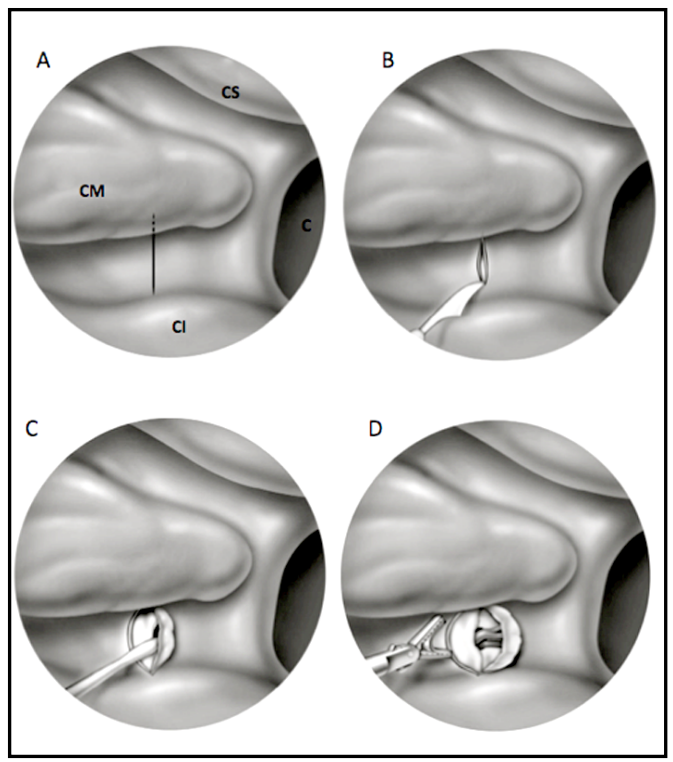

Figura 1. A) Visión endoscópica quirúrgica. Se aprecia la cola del cornete medio (CM), porción posterior de cornete superior (CS), porción posterior de cornete inferior $(\mathrm{Cl}$ ) y coana (C). B) Se realiza una incisión mucosa en forma de "L" $1 \mathrm{~cm}$ anterior a la inserción posterior de la cola del cornete medio, continuando hasta la inserción del cornete inferior. C) Se levanta un flap mucoperióstico posterosuperior hasta encontrar el foramen de la AEP. D) Se procede a ligar la AEP con clips mediante visión directa. Luego el flap mucoperióstico es vuelto a su posición original. Modificado de Von Buchwald et al, Operative Techniques in Otolaryngology 2006; 17: 28-30. 
(HTA) en tratamiento y $26 \%$ tenía historia de consumo de dosis de aspirina de al menos $75 \mathrm{mg}$ en la semana previa al diagnóstico de epistaxis. Como otro antecedente, $47,8 \%$ de los pacientes tenía historia de al menos un episodio anterior de epistaxis (Tabla 1).

El $56,5 \%$ de estos pacientes ingresó al hospital desde el Servicio de Urgencia del HCUCh, mientras que el $43,5 \%$ restante, fue hospitalizado desde el consultorio externo de especialidad del servicio. Los cuadros de epistaxis fueron unilaterales, derecho 0 izquierdo, en $91,3 \%$ de los casos, siendo $8,7 \%$ bilaterales. La hemoglobina promedio al ingreso fue de 12,4 $\pm 2,4 \mathrm{~g} / \mathrm{dL}$. Todos los pacientes fueron tratados inicialmente con taponamiento nasal prequirúrgico, el cual fue de tipo anterior en 3 casos, con una duración promedio de $38 \pm 12$ hrs. y de tipo posterior más anterior en los restantes 20 casos con una duración de $124 \pm 27$ hrs. como promedio (Tabla 2).

\section{Manejo quirúrgico}

El promedio de tiempo entre el ingreso al hospital y la resolución quirúrgica del cuadro de epistaxis fue de $87,8 \pm 27 \mathrm{hrs}$. (rango entre 12 y $360 \mathrm{hrs}$.). Con respecto al método de control de epistaxis, se realizó ligadura con clips más electrocauterización de AEP en 52,1\% de los casos, ligadura con clips de AEP en $26,2 \%$, y electrocauterización aislada de AEP en $21,7 \%$ de los casos. Se complementó la técnica quirúrgica con la realización de uncinectomía en 17,3\%, ligadura por clips de arterias etmoidales en $13 \%$ de los casos y una antrostomía en $13 \%$ de los casos. El tiempo quirúrgico fue de 101ะ52 minutos de duración en promedio (rango entre 45 y 180 minutos). El tiempo de estadía hospitalaria fue de 4,4 $\pm 3,6$ días en promedio (rango entre 1 y 12 días) (Tabla 2).

\section{Evolución posquirúrgica y costos}

El mantenimiento del taponamiento nasal posquirúrgico fue de $5,2 \pm 4,2$ días en promedio. En cuanto a las complicaciones registradas en el posoperatorio, éstas ocurrieron en 4 casos $(17 \%)$, y correspondieron a sangrado que resolvió en forma espontánea en 2 de los casos, sangrado que requirió de nuevo taponamiento en uno de los casos y epistaxis recidivante que requirió nueva ligadura de AEP en el posoperatorio en el último de los casos.

\section{DISCUSIÓN}

En la última década se ha experimentado una significativa expansión en alternativas para el manejo de las epistaxis. Si bien es cierto, las tradicionales técnicas de taponamiento nasal aún se realizan con éxito, se han incorporado al tratamiento nuevas tecnologías como el uso de materiales de tipo coloides para taponamiento, el uso de endoscopía, fibra óptica, láser y técnicas de embolización selectiva. En el presente estudio se describió la experiencia del HCUCh en el manejo endoscópico de pacientes con epistaxis posterior entre los años 2007 y 2011.

Con respecto a las comorbilidades de los pacientes, cabe destacar el antecedente de HTA en el $60 \%$ de los pacientes, que de acuerdo a lo descrito en la

Tabla 1. Características clínicas generales de los pacientes con epistaxis posterior tratados endoscópicamente

\begin{tabular}{|c|c|}
\hline \multicolumn{2}{|l|}{ Características de los pacientes } \\
\hline Número de pacientes & 23 \\
\hline Número de ingresos & 24 \\
\hline Edad (años \pm DE) [rango] & $52 \pm 18[16-81]$ \\
\hline \multicolumn{2}{|l|}{ Sexo } \\
\hline Masculino & $15(65,2 \%)$ \\
\hline Femenino & $8(34,8 \%)$ \\
\hline Antecedentes epistaxis antigua & $11(47,8 \%)$ \\
\hline \multicolumn{2}{|l|}{ Comorbilidades y fármacos } \\
\hline HTA en tratamiento & $14(60 \%)$ \\
\hline Uso de aspirina & $6(26 \%)$ \\
\hline Presión arterial al ingreso $( \pm D E)$ & $154 \pm 32 / 88 \pm 17 \mathrm{~mm} \mathrm{Hg}$ \\
\hline \multicolumn{2}{|l|}{ Hemoglobina y plaquetas al ingreso } \\
\hline $\mathrm{Hb}(\mathrm{g} / \mathrm{dL})( \pm \mathrm{DE})[\mathrm{rango}]$ & $12,4 \pm 2,4 \mathrm{~g} / \mathrm{dL}[6,8-16,4]$ \\
\hline Plaquetas $\left(\mathrm{mm}^{3}\right)( \pm \mathrm{DE})$ & $238.000 \pm 74.000$ \\
\hline
\end{tabular}


Tabla 2. Características prequirúrgicas de las epistaxis y descripción de procedimientos realizados

\begin{tabular}{|lcc|}
\hline Características & Número & $(\%)$ \\
\hline Taponamiento nasal preoperatorio & & \\
$\quad$ Anterior & 3 & $(13)$ \\
Posterior más anterior & 20 & $(87)$ \\
Ingreso al Hospital & & $(56,5)$ \\
$\quad$ Servicio de urgencia & 13 & $(43,5)$ \\
$\quad$ Policlínico ORL & 10 & $(56,5)$ \\
AEP tratada & 13 & $(34,7)$ \\
$\quad$ Derecha & 8 & $(8,8)$ \\
Izquierda & 2 & $(21,7)$ \\
Bilateral & & $(26,2)$ \\
Técnica quirúrgica utilizada & 5 & $(52,1)$ \\
$\quad$ Cauterización bipolar AEP & 6 & $(17,3)$ \\
$\quad$ Ligadura AEP con clips & 12 & $(13)$ \\
Técnica combinada & & $(13)$ \\
Procedimientos complementarios & 4 & 3 \\
$\quad$ Uncinectomía & 3 & \\
Antrostomía & & \\
Ligadura arterias etmoidales & 3 & \\
\hline
\end{tabular}

literatura ${ }^{5,11}$, constituye una patología crónica frecuentemente presente en pacientes con epistaxis, que puede determinar complicaciones como resangrado en el posoperatorio, por lo que se hace necesario su adecuado control. En el estudio de Reza y cols $^{12}$, se identificó como factores de riesgo aislado de resangrado precoz (primeras 2 semanas) en el posoperatorio, un recuento bajo de plaquetas al ingreso (p 0,03), y la no utilización de electrocauterización en el manejo endoscópico ( $p$ 0,02). Tanto la HTA como el uso crónico de aspirina no constituyeron factores de riesgo aislado según este estudio.

Inicialmente el equipamiento quirúrgico con que contaba el servicio no incluía instrumental adecuado para ligar AEP 0 arterias etmoidales, razón por la cual en las primeras cirugías se realizó electrocauterización de AEP, adquiriendo posteriormente una clipera apropiada y cauterizador bipolar de adecuada maniobrabilidad con que se realizaron el resto de los procedimientos.

El estudio de Klotz y cols ${ }^{9}$, comparó en 203 pacientes el tratamiento quirúrgico (ligadura arterial de maxilar interna o $\mathrm{AEP}$ ) con el no quirúrgico (taponamiento antero-posterior o embolización), mostrando una tasa de éxito del $90 \%$ para los procedimientos quirúrgicos, del $75 \%$ para la embolización y del $62 \%$ para el taponamiento anteroposterior, con una estancia hospitalaria superior en los pacientes tratados médicamente (5,29 días) que en los de manejo quirúrgico (2,1 días).
En nuestra serie de pacientes, sólo existieron 2 casos $(8,7 \%)$ de pacientes con antecedentes de HTA de difícil manejo, que presentaron resangrado en el posoperatorio y que requirieron taponamiento 0 una nueva ligadura de AEP para su control, por lo que la tasa de éxito lograda con técnica endoscópica fue de $91,3 \%$ en nuestro centro. El tiempo de estadía hospitalaria de nuestra serie de pacientes fue de 4,43 $\pm 3,6$ días en promedio. Cabe mencionar que uno de los pacientes que presentó una estadía prolongada presentaba compromiso hemodinámico al ingreso, por lo que requirió múltiples transfusiones y una estadía posoperatoria en intermedio quirúrgico.

Otro factor importante a la hora de decidir el manejo de un paciente con epistaxis, es la disponibilidad de pabellón con el equipamiento correcto (torre para endoscopía), lo cual constituye un impedimento real actual, que muchas veces limita la posibilidad de realizar el manejo de una epistaxis de urgencia bajo esta técnica.

\section{CONCLUSIÓN}

El manejo endoscópico de epistaxis posterior mediante ligadura o cauterización de AEP constituye una alternativa eficaz de tratamiento en estos pacientes. Presenta una tasa de fracaso, descrita en 
la literatura, similar a la de otras técnicas y estos casos pueden ser resueltos mediante diversas alternativas como la embolización selectiva arterial.

Una de las grandes ventajas del manejo endoscópico de las epistaxis, es su baja tasa de complicaciones, lo que reduce la morbilidad para el paciente y determina un menor costo por días de hospitalización. Corresponde a una técnica reproducible, relativamente sencilla de enseñar y realizar, que requiere de equipamiento o instrumental quirúrgico disponible en la mayoría de los centros formadores de especialistas. Sin embargo se debe tener en consideración contar con una buena evaluación prequirúrgica multidisciplinaria en estos pacientes, a manera de evitar las complicaciones que, aunque escasas, pueden presentarse.

Esperamos que a futuro se desarrollen protocolos para el tratamiento de epistaxis posteriores, donde el manejo endoscópico sea incorporado como una herramienta segura y eficiente para estos pacientes.

\section{BIBLIOGRAFÍA}

1. Naser A, Aedo C. Epistaxis: diagnóstico y alternativas terapéuticas actuales. Rev Hosp Clin Univ Chile2007; 18; 227-38.

2. Viehweg L, Roberson B, Hudson J. Epistaxis: diagnosis and treatment. J Oral Maxillofac Surg 2006; 64: 511-8.

3. Strong B, Johnson P, Bell A. Intractable epistaxis: transantral ligation vs. embolization: efficacy review and cost analysis. Otolaryngol Head Neck Surg 1995; 113: 674-8.

4. Cullen M, TAmi T. Comparison of internal maxillary artery ligation versus embolization for refractory posterior epistaxis. Otolaryngol Head and Neck Surg 1998; 118: 636-42.

5. Wang L, Vogel D. Posterior epistaxis: comparison of treatment. Otolaryngol Head and Neck Surg 1981; 89: 1001-6.

6. WOOLFORD J, JONES S. Endoscopic ligation of anterior ethmoidal artery in treatment of epistaxis. $J$ Laryngol Otol 2000; 114: 858-60.

7. Douglas A, Gupta D. Endoscopic assisted external approach anterior ethmoidal artery ligation for the management of epistaxis. $J$
Laryngol Otol 2003; 117: 132-3.

8. Ahmed A, Woolford T. Endoscopic bipolar diathermy in the management of epistaxis: an effective and costefficient treatment. Clin Otolaryngo/2003; 28: 273-5.

9. Klotz M, Darrell A, Mark R. Surgical Management of Posterior Epistaxis: A Changing Paradigm. Laryngoscope 2002; 112: 1577-82.

10. VOEGELSR. Endoscopic ligature of the sphenopalatine artery for severe posterior epistaxis. Otolaryngol Head Neck Surg 2001; 124: 464-7.

11. Thornton M, Mahesh B. Posterior Epistaxis: Identification of Common Bleeding Sites. Laryngoscope 2005; 115: 588-90.

12. Reza N, TAREQ M, Hauloff D. Outcome of Endoscopic Sphenopalatine Artery Occlusion for Intractable Epistaxis: A 10-Year Experience. Laryngoscope 2007; 117: 1452-6.

13. Jguss J, Marc A. Hard Palate Necrosis After Bilateral Internal Maxillary Artery Embolization for Epistaxis. Laryngoscope 2007; 117: 1683-4.

14. Francini G, Voegels R. Severe Posterior EpistaxisEndoscopic Surgical Anatomy. Laryngoscope 2008; 118: 156-61.

15. Goddard J, Reiter E. Inpatient management of epistaxis: Outcomes and cost. Otolaryngol Head Neck Surg 2005; 132: 707-12.

16. ChIU T, McGarry G. Prospective clinical study of bleeding sites in idiopathic adult posterior epistaxis. Otolaryngol Head Neck Surg 2007; 137: 390-3.

17. StankiEWICZ J. Nasal endoscopy and control of epistaxis. Curr Opin Otolaryngol Head Neck Surg 2004; $12:$ 43-5.

18. Vergara J, Hernández E. Comparación del manejo quirúrgico versus no quirúrgico en pacientes con epistaxis posterior. Acta Otorrinolaringol Esp 2006; 57: 41-6.

19. WIOROWSKI M, PHILIPPE S. Indications and results of cauterization by endoscopic approach of the sphenopalatine artery in severe posterior epistaxis. Auris Nasus Larynx 31 2004; 98: 131-3.

20. Jameson J, CAVE D. Hormonal and Antihormonal Therapy for Epistaxis in Hereditary Hemorrhagic Telangiectasia. Laryngoscope 2004; 114: 705-9.

21. Von Buchwald C, Tranum-Jensen J. Endoscopic sphenopalatine artery ligation or diathermy. Operative Techniques in Otolaryngology 2006; 17: 28-30. 